\title{
Klimawandel und mehr: aktuelle Themen in „Raumforschung und Raumordnung"6
}

\author{
Andreas Klee $\cdot$ Johannes Schulte $\cdot$ Hendrikje Wehnert
}

(C) Springer-Verlag 2012

Der Klimawandel ist schon seit längerer Zeit ein Thema, das in Wissenschaft und Politik immer wieder aufgegriffen wird. Die globale Erderwärmung scheint unaufhaltbar und macht ein Handeln über politische Grenzen hinweg notwendig. Das Ausmaß der Erwärmung und ihre Konsequenzen sind indes noch unsicher, verschiedene Szenarien weichen stark voneinander ab und reichen von einer grundpessimistischen bis zu einer optimistischen, teils ignoranten Prognose. Fest steht, dass sich das Klima verändert und sich somit die Lebensverhältnisse in allen Gebieten der Erde mitverändern. Ob Klimaschutz oder Anpassung an die Folgen des Klimawandels: Es ist auch die Aufgabe der Raum- und Stadtplanung, sich auf diese neuen Verhältnisse einzustellen und entsprechend zu reagieren. Auf globaler Ebene wurde im Rahmenübereinkommen der Vereinten Nationen über Klimaänderung bereits 1992 die Problematik erkannt. Mit dem Zusatz des Kyoto-Protokolls von 1997 wurde der Klimaschutz für die teilnehmenden Industrieländer festgeschrieben, die Implementierung bereitet jedoch vielen Staaten Probleme. Auch wenn auf supranationaler Ebene Rahmenbedingungen besprochen werden, müssen diese Top-down kommuniziert werden, denn sie entfalten

\footnotetext{
Dr. A. Klee $(\bowtie) \cdot J$. Schulte

Akademie für Raumforschung und Landesplanung - LeibnizForum für Raumwissenschaften, Hohenzollernstraße 11, 30161 Hannover, Deutschland

E-Mail: klee@arl-net.de

J. Schulte

E-Mail: j-schulte@arcor.de

H. Wehnert

Leibniz-Institut für ökologische Raumentwicklung,

Weberplatz 1, 01217 Dresden, Deutschland

E-Mail: h.wehnert@ioer.de
}

auf nationaler, regionaler und kommunaler Ebene zum Teil erhebliche Wirkungen.

In diesem Heft der Zeitschrift „Raumforschung und Raumordnung“werden zwei Artikel zur Anpassung an die Folgen des Klimawandels vorgestellt. Stefanie Baasch, Sybille Bauriedl, Simone Hafner und Sandra Weidlich untersuchen die Anpassungsmöglichkeiten auf regionaler Ebene. Sie weisen auf differenziert ausgeprägte Governance-Strukturen hin und sehen gerade in der Anpassung an die Folgen des Klimawandels die Problematik, dass sich keine Maßnahmen von oben herab durchsetzen lassen. In ihrer Zusammenfassung der Herausforderungen für Klimawandel-Governance sind die Autorinnen der Ansicht, dass es stets maßgeschneiderter Maßnahmen bedarf, die möglichst auf kleinräumiger Ebene umgesetzt werden sollten. Hierzu bietet sich die Regionalplanung als Instrument an. Aus Ergebnissen des Verbundprojekts KLIMZUG-Nordhessen werden abgeleitete Optimierungsversuche formuliert. Für die Raum- und Regionalplanung sowie für die Forst- und Landwirtschaft werden Institutionen, Steuerungsmodi und Instrumente analysiert, die mögliche Anpassungsmaßnahmen begleiten können.

Sonja Deppisch und Meike Albers gehen in ihrem Artikel darauf ein, welchen Beitrag bzw. Mehrwert transnationale Kooperationen bei der Anpassung an den Klimawandel leisten können. Betont wird hierbei die starke Raumwirksamkeit des Klimawandels, die der Raumplanung eine zentrale Rolle zuweist. Eine effiziente Aufgabenteilung von der transnationalen bis zur lokalen Ebene ist hierbei notwendig. Regionale Auswirkungen wie erhöhte Wintertemperaturen oder sich ändernde Niederschläge werden anhand von drei Fallstudien im Ostseeraum dargestellt. In den Stadtregionen Stockholm und Rostock wurden unterschiedliche Maßnahmen zur Anpassung an die Folgen des Klimawandels bereits in Instrumente der räumlichen Planung integriert. Durch 
transnationale Kooperationen und stetigen Erfahrungsaustausch - auch im Rahmen von makroregionalen Strategien - kann sich ein additiver Nutzen ergeben, der es vielen lokalen Akteuren ermöglicht, effektive Maßnahmen einzuleiten. Denn wie die Autorinnen hervorheben, sind Kommunen meist noch unerfahren mit Anpassungsmaßnahmen und dadurch oftmals überfordert, zudem tendieren sie zu lediglich kurzfristigem Handeln. Die Regionalplanung kann hier ein adäquates, intermediäres und richtungsweisendes Mittel sein, um Kommunen zu entlasten. Kooperationen und Aufgabenteilung sollen auf allen Ebenen einen Mehrwert generieren und Planungen sinnvoll ergänzen.

Anna Growe geht in ihrem Beitrag auf den räumlichen Kontext von Wissen als Produktionsfaktor ökonomischer Prozesse ein. Sie untersucht Raummuster unterschiedlicher Wissensformen und stellt dabei den Einfluss von Transaktionskosten auf Konzentrationsprozesse wissensintensiver Dienstleister im deutschen Städtesystem dar. Lange Zeit wurde Wissen als Produktionsfaktor ausgeblendet und in Raumentwicklungstheorien als exogen gegeben angenommen. Die Autorin ist von der großen Bedeutung von Wissen als Produktionsfaktor überzeugt und liefert in ihrem Beitrag empirische Belege dafür, dass die Produktion von wissensintensiven Dienstleistungen nicht bei allen Wissensformen Prozesse der Konzentration initiiert. Letztlich ist die räumliche Nähe für alle Wissensformen ein wichtiger Faktor, da sie Transaktionskosten reduziert.
Auf die Bedeutung räumlicher Nähe gehen auch Jean Peyrony und Olivier Denert ein. Sie berichten aus ihrer Forschung zur Planung in französischen Grenzräumen. Ausgehend von der Feststellung, dass in vielen Grenzräumen staatenübergreifende, raumbezogene Informationen fehlen, untersuchen die Autoren deren Funktion bei der räumlichen Planung, da sie eine wichtige Grundlage für eine Zusammenarbeit über Grenzen hinweg darstellen können. Die Autoren gehen dabei näher auf das Konzept des „Grenzraums" am Beispiel der Grenzregion Frankreich-Luxemburg ein, zeigen die unterschiedlichen Maßstabsebenen auf und stellen fest, welche Werkzeuge zur Verfügung stehen. Klar verdeutlichen sie die Notwendigkeit eines integrierten Entwicklungsansatzes sowie einer grenzüberschreitenden, mehrere Ebenen umfassenden Governance in Grenzregionen, um sie nachhaltig zu stärken.

Wir freuen uns, unseren Leserinnen und Lesern wieder einmal einen englischsprachigen Beitrag anbieten zu können. Auch das nächste Heft von „Raumforschung und Raumordnung" wird sich der Sprache der internationalen Wissenschaft bedienen, wenn im Rahmen eines Schwerpunktheftes in zahlreichen Beiträgen das Thema „Vulnerability and Resilience from a socio-spatial perspective" beleuchtet wird.

Wir wünschen unseren Leserinnen und Lesern wie immer eine spannende und erkenntnisreiche Lektüre. 